\title{
El desarrollo como metarrelato de la modernidad
}

\author{
Bravo, Olga* \\ Marín González, Freddy**
}

\section{Resumen}

El presente artículo constituye un avance del proceso de construcción teórica del proyecto de tesis: "Red de innovación productiva como modelo de organización para el desarrollo local sostenible", el cual analiza mediante una revisión bibliográfica y documental, la conceptualización del desarrollo en el contexto de la crisis de los fundamentos y valores de la modernidad. Partiendo de la noción de progreso como génesis de la idea de desarrollo surgida en la modernidad, se discuten las modificaciones conceptuales ocurridas en la megamodernidad, que ha dado lugar a una sociedad del riesgo caracterizada por graves problemas sociales, económicos, políticos y ecológicos de alcance global. Se concluye que desde una visión sistémica, multidimensional y compleja bajo una dialéctica global-local, la sociedad sostenible sólo puede construirse con base en la capacidad del sistema social de coevolucionar, manteniendo su integridad, con su sistema ambiental cambiante, el estudio de cuyos procesos conlleva abordajes interdisciplinarios y transdisciplinarios.

Palabras clave: Desarrollo sostenible, modernidad, megamodernidad.

\section{Development as a Meta Story of Modernity}

\begin{abstract}
This article constitutes an advance in the theoretical construction process for the thesis project, "Network for Productive Innovation as an Organization Model for Local Sustainable Development, "which analyzes, through a bibliographic and documentary review, the conceptualization of development in the context of the crisis in the foundations and values of modernity. Starting from the notion of progress as the genesis for the idea of development that arose in modernity, the conceptual
\end{abstract}

\section{Recibido: 20-04-11. Aceptado: 23-11-11}

* Magister Scienciarum en Microbiología. Cursante Doctorado en Planificación y Gestión del Desarrollo Regional (LUZ-Núcleo Punto Fijo). e-mail: olgabravo10@gmail.com

** Doctor en Ciencias Humanas. Profesor Titular, LUZ- Núcleo Punto Fijo. e-mail: freddyvmaring@gmail.com 
modifications that occurred in mega-modernity are discussed, which have given way to a society of risk, characterized by serious social, economic, political and ecological problems of a global scope. Conclusions are that from a systemic, multidimensional and complex vision under a global-local dialectic, the sustainable society can only construct itself based on the capacity of the social system to co-evolve, maintaining its integrity, with its changing environmental system, the study of whose processes carry with it interdisciplinary and trans-disciplinary approaches.

Keywords: Sustainable development, modernity, mega-modernity.

\section{Introducción}

Una de las discusiones teóricas más importantes en las ciencias sociales, iniciada durante el último tercio del siglo $X X$, concierne a la conceptualización de la forma en que actualmente se desenvuelven los sistemas sociales, económicos, políticos y culturales, si se corresponde efectivamente con un salto a la postmodernidad, una desviación del proyecto original de la modernidad, o su profundización y radicalización, esto es, la megamodernidad. Como parte consustancial de esta controversia, se produce también un cambio en la percepción fenomenológica del desarrollo, dando lugar a nuevas interpretaciones que incorporan las dimensiones territorial, social y ambiental, y contribuyen a un pensamiento económico que reconoce sus vínculos indisolubles con el orden social subyacente, relativizando la posición del sistema económico en el conjunto de la estructura social.

Al respecto, el enfoque socio-territorial del desarrollo se deslastró de la noción de objeto para emerger como sujeto y actor principal de su trama, pero el enfoque ambiental avanzó sin un cuestionamiento profundo de la concepción de desarrollo forjada en el contexto de la modernidad, es decir, aquella que lo identifi- ca con crecimiento económico, y en la cual la asunción de la naturaleza como simple proveedora, como recurso infinito a explotar -capaz de soportar todas las cargas que le impongan las actividades humanas- ha llevado a una dicotomía sociedad-naturaleza artificial y destructiva. No obstante, la evidente transición de las complejas sociedades actuales hacia una sociedad global, caracterizada por peligrosos "riesgos" de alcance cosmopolita, que afectan su viabilidad y sostenibilidad, acarrea una fuerte crítica de algunos presupuestos fundamentales de la episteme de la modernidad.

Jean-Francois Lyotard (1987), defiende la idea de que el proyecto de la modernidad se encuentra agotado, o más bien ha sido reemplazado por los éxitos del mercado, de modo que se asiste a la "postmodernidad"; para Jürgen Habermas $(1989,1992)$ la modernidad es un "proyecto inacabado", cuya actual patología no es inherente a sí mismo sino a desviaciones en su desarrollo; mientras que Anthony Giddens $(1994,2002)$ lidera el enfoque de que los actuales cambios son sólo "consecuencias de la modernidad". Por lo cual, cabe preguntar ¿se encuentran en crisis los fundamentos y valores de la modernidad y, en consecuencia, la conceptualización del desarrollo como uno de sus grandes metarrelatos? 


\section{Los fundamentos de la modernidad}

La modernidad, de acuerdo con Beltran y Cardona (2005), es el nuevo orden social que substituyó el régimen feudal, a partir del siglo XVIII, como resultado de un largo proceso histórico de carácter global, abonado por acontecimientos religiosos (reforma luterana), socio-políticos (revolución francesa), intelectuales (ilustración), y tecnológicos (revolución industrial), que trastocaron el orden existente y abrieron las puertas al cambio social. Tales acontecimientos propiciaron el tránsito hacia la modernidad, bajo el impulso de cuatro grandes ideas, relacionadas entre sí:

- Ruptura con el principio trascendente de ordenamiento de la sociedad, que cuestiona las bases del régimen premoderno sustentado en la existencia de un principio divino (concepción teocéntrica) que organiza y orienta dicha sociedad.

- Búsqueda de un principio inmanente, puesto que el hombre aparece como el principio del orden (concepción antropocéntrica) que no sólo busca intereses privados sino que es un agente moral, capaz de construir una convivencia pública, trasladando a la política la función integradora que cumplía la religión.

- Absolutización del concepto de razón y progreso, dado que el acelerado proceso de urbanización e industrialización fue promovido por una ilimitada confianza en las posibilidades de la ciencia y la razón, así como por la idea de historia como progreso, entendido éste como crecimiento continuo, posible a partir del cada vez mayor dominio del hombre sobre la naturaleza.

- Conciencia de ruptura con el pasado, en tanto se erige una concepción liberadora, crítica y reflexiva, enfrentada a las formas tradicionales de organización social, que pugna por crear un mundo nuevo y un hombre nuevo.

Al respecto, Carretero (2002) señala que la modernidad introduce una filosofía de la historia que gravita sobre la idea de que la humanidad avanza hacia un estado futuro de perfección que es preciso conquistar, noción que exhibe una doble vertiente complementaria. Por una parte, a través de un desarrollo científico-tecnológico, perseguirá un dominio ilimitado de la naturaleza que se identifica, de modo reduccionista, con un mayor bienestar social y, por otra parte, amparándose en el pensamiento ilustrado, busca un mayor grado de perfección para la humanidad, mediante el primado de la razón en su lucha contra los prejuicios y religiones. No obstante, para el autor, más que una discontinuidad histórico-cultural con respecto a la tradición judeo-cristiana, la modernidad fue una secularización de los mismos principios doctrinales, ya que la idea de progreso en el fondo es la misma que aquella de la salvación mesiánica, pues ambas descansan sobre una visión lineal de la historia.

Por su parte, Berman (1991) divide la historia de la modernidad en tres fases. En la primera (inicios del siglo XVI hasta finales del XVIII), las personas comienzan a experimentar la vida moderna, aunque tienen poca o ninguna percepción de ello. La segunda fase se inicia con la gran 
ola revolucionaria francesa de la década de 1790 , cuando la gente comparte la sensación de estar viviendo una época nueva en todas las dimensiones de su vida, de estar viviendo simultáneamente en dos mundos: dicotomía desde la cual emergen y se despliegan las ideas de "modernización" y "modernismo". La tercera fase ocurre en el siglo XX cuando el proceso de modernización se expande para abarcar prácticamente todo el mundo y la idea de la modernidad, concebida en numerosas formas fragmentarias, se va extendiendo lentamente, de manera desigual, discontinua y conflictiva, desde la Europa Occidental hasta configurar hoy una edad moderna que ha perdido el contacto con las raíces de su propia modernidad.

Berman llama "modernización" a los procesos históricos de formación de la civilización moderna: descubrimientos de las ciencias físicas que modificaron la percepción del universo; industrialización asociada a la transformación del conocimiento en tecnología; crecimiento urbano, migraciones internas, declive del mundo rural y creación de nuevos espacios de integración social más amplios (la ciudad y el Estado-nación); sistemas de comunicación de masas; ampliación de intercambios económicos hasta configurar mercados nacionales y el mercado capitalista mundial. $Y$ denomina "modernismo" a los valores y cosmovisiones que los han acompañado, subrayando su ambivalencia y contradicciones: el cambio incesante, la transformación del mundo cerrado tradicional en un mundo abierto, pleno de posibilidades pero también de desorientación, inestabilidad e inseguridad.
Con respecto a este doble ideario presente en el advenimiento de la sociedad moderna, Lechner (1999) relaciona la "modernización" con una racionalidad instrumental, o sea el cálculo medio-fines, cuyas expresiones máximas son el mercado y la burocracia; mientras que el "modernismo" se vincula con una racionalidad normativa, a través de la institución de valores, normas y representaciones del orden social, cuya expresión máxima es la democracia; de modo que la tensión aparece porque si bien la modernidad es la expresión de principios universalistas de legitimación, la modernización aparece como la gran fuerza universalista.

Beltrán y Cardona (2005) señalan que en cuanto la sociedad moderna toma cuerpo el proyecto liberador se va vaciando de contenido, reemplazando la razón emancipadora y crítica por una razón instrumental que somete al hombre, operándose un divorcio entre los dos componentes indisolubles de la modernidad, pues ésta se convierte en la ideología que avala, exalta y defiende la modernización. En el caso de América Latina y otros continentes subdesarrollados, donde no hubo una experiencia cultural de modernización -el capitalismo de mercado no es un dato histórico- sino que ha sido el Estado quien se ha arrogado la tarea de instaurar una sociedad moderna, las culturas tradicionales resultaron avasalladas por la dinámica del mercado mundial, transmutándose en lo que Lechner (1999) denomina una "modernización sin modernidad", que limita las posibilidades de un desarrollo humano sustentable.

Los planteamientos expuestos permiten evidenciar, por una parte, que la 
idea de "desarrollo" hunde sus raíces en la noción de "progreso" surgida en la modernidad, dentro de una concepción lineal de la historia que supone el avance continuo de la humanidad hacia un estado futuro de bienestar material y perfección moral. Metarrelato ideológico sustentado en las inmensas posibilidades de la ciencia, para el dominio ilimitado de la naturaleza, y la razón, cuyo primado facilitaría la comprensión y construcción de un mundo mejor.

Por otra parte, el término desarrollo comienza a ser utilizado a mediados del siglo XX, durante la llamada tercera etapa de la modernidad, cuando convergen el auge pleno de los procesos históricos de la modernización y su progresivo vaciamiento de los valores y cosmovisiones de la modernidad, por lo que su conceptualización se encontrará cada vez más referida al crecimiento económico, al uso indiscriminado de los recursos de la naturaleza, a la identificación del consumo con el bienestar y a la potenciación del desarrollo tecnológico, al margen de la dimensión humana y social.

\section{3. ¿Postmodernidad o megamodernidad?}

Desde la década de 1970 se evidenció la crisis de los ideales de la modernidad bajo el peso de las fuerzas de la modernización, generando una polémica en torno a la postmodernidad, cuyo núcleo es la reactualización o superación del pensamiento de la llustración, esto es, si se deben revivir las intenciones del iluminismo europeo o reconocer que todo el proyecto de la modernidad es una causa perdida. Con otras palabras, si se han agotado hasta sus últimas consecuencias las posibilidades de la razón moderna, o si el modelo declina y se requieren nuevas corrientes paradigmáticas

Lyotard (1987) toma partido por la segunda opción: el proyecto de la llustración ha quedado clausurado, y designa con el término de postmodernidad al estado de la cultura después de las transformaciones que han afectado las reglas de juego de la ciencia, la literatura y las artes a partir del siglo XIX. Considera que se ha entrado en una nueva época signada por la desaparición de los "grandes discursos", en particular, el discurso del progreso que -tanto en la ideología marxista como en la capitalista- intentaba dar un sentido a la marcha de la historia, y el de la ciencia, basada a su vez en un metadiscurso justificador -la filosofía- mediante el cual se validan sus enunciados como un "valor de verdad". La postmodernidad se encuentra entonces referida al reinado absoluto de la ciencia y la razón, a la incredulidad en torno a los metarrelatos ideológicos (filosóficos y ético-políticos) de legitimación del progreso, que fijó una orientación hacia el porvenir a las sociedades occidentales desde el siglo XIX.

En una posición contraria a la corriente postmoderniista, Habermas (1989) plantea que la modernidad es un proyecto a perfeccionar, sumido en ciertos escollos de los cuales es preciso salir. Considera que tal proyecto estuvo basado en el desarrollo de una ciencia objetiva, una moral universal, una ley y un arte autónomos, regulados por lógicas propias, por lo cual pudieron institucionalizarse el discurso científico, las teorías morales, la jurisprudencia, y la producción y crítica de arte. En consecuencia, se 
fundamentó en la esperanza de que la ciencia, la moral y el arte promovieran no sólo el control de las fuerzas naturales sino también la comprensión del mundo y del individuo, el progreso moral, la justicia de las instituciones y la felicidad de los hombres.

Sin embargo, esta diferenciación de cada uno de los dominios de la cultura, desembocó en la autonomía de segmentos manipulados por especialistas y escindidos de la hermenéutica de la comunicación diaria, o sea, lo que se incorpora a la cultura a través de la reflexión y la práctica especializadas no enriquece necesaria ni inmediatamente la organización racional de la cotidianeidad social, ya que el proceso de comunicación necesita de una tradición cultural que cubra todas las esferas complementarias del saber (cognitivo-instrumental, moral-práctica y estético-expresiva).

Esto significa, según Habermas (1992), que en el mundo actual ha crecido la distancia entre la cultura de los expertos y la del público en general, y entre los intereses del "sistema" (el capitalismo de mercado, las instituciones políticas y sociales) y "el mundo de la vida" (la ciudadanía). Por lo tanto, para culminar el proyecto de la modernidad, se requiere un modelo por el cual se produzca una definitiva secularización del hombre, junto con su emancipación de las fuerzas alienantes del sistema establecido. Este modelo es la comunidad ideal de comunicación en la que se sustituye la filosofía del sujeto (razón fundamentada en sí misma), por la razón comunicativa, o sea, por el consenso racional adquirido tras el debate, en que los participantes se encuentran libres de coacciones internas o externas.
Giddens (1994) critica el enfoque de Lyotard como una visión muy parcial de la época actual, ya que la modernidad no puede reducirse a una lógica única -económica, política o cultural- sino que supone la articulación de esas lógicas. En su perspectiva, la sociedad moderna es un sistema integrado multidimensional, movido por la influencia de un complejo de instituciones (capitalismo, industrialización, estados-nación, e individualismo), que han transformado el mundo durante los últimos tres siglos, y configurado un fenómeno mundial de radicalización de la modernidad, en la cual se experimenta una transición hacia una sociedad cosmopolita global, impulsada por las fuerzas del mercado, los cambios tecnológicos y los cambios culturales.

Giddens describe la modernidad yuxtaponiendo cuatro dimensiones institucionales, todas constitutivas: capitalismo (sistema de producción centrado en la relación entre la propiedad privada del capital y la mano de obra asalariada); vigilancia (control político de la información y supervisión de las actividades de la población); poder militar (control de los medios de violencia en el contexto de la industrialización y la guerra); e industrialismo (alianza entre ciencia y tecnología para la apropiación de los recursos de la naturaleza). El desenvolvimiento de estas dimensiones habría provocado la aparición de efectos contradictorios, ya que el propio avance de la sociedad industrial conlleva el inevitable surgimiento de nuevos y terribles "riesgos", derivados del colapso de los mecanismos de control económico, el crecimiento de un poder totalitario discursivamente hegemónico, la posibilidad de un conflicto nuclear a gran 
escala, y el desastre ecológico, que amenaza con la destrucción de la vida.

Además, Giddens (2002) se enfoca en la comprensión de los mecanismos de auto-identidad constituidos por las instituciones de la modernidad y que a su vez influyen en su constitución, esto es, dado que los individuos participan en las influencias sociales al forjar sus auto-identidades, estas influencias -que pueden ser de carácter local en el contexto específico de la acción- se manifiestan como globales en sus consecuencias e implicaciones. Por tanto, es imposible disociar la constitución de las sociedades modernas actuales del impacto dramático de la globalización o de los riesgos sociales, tanto sobre el individuo como sobre la colectividad, que afecta los aspectos más personales de su existencia y amerita la evolución de un "sujeto reflexivo" capaz de auto-confrontar las peligrosas consecuencias de la modernidad.

En concordancia, Beck (1994, 2003) plantea la transición del estado-nación a una "sociedad postnacional" y de la sociedad industrial a una sociedad postindustrial, o "sociedad del riesgo". El estado-nación -principal estamento político de la modernidad- encargado del bienestar público, ha dado paso a la sociedad postnacional, globalizada desde el interior, en la cual se desacopla la equiparación de territorio, sociedad e identidad política, disolviendo jurisdicciones y fronteras regionales. Entre tanto, la sociedad industrial evoluciona hacia la sociedad del riesgo, en la que la producción de riesgos y la individualización se convierten en los procesos sociales predominantes, una nueva fase de la modernidad caracterizada por su reflexividad, es decir, el proceso de modernización -al tratar de hacer frente a los riesgos- se torna reflexivo y comienza a cuestionar a las propias instituciones sobre las que se ha asentado: la ciencia, el progreso o el industrialismo, son puestos en duda en esta fase de la modernidad.

La sociedad del riesgo formulada por Beck, cuyos inicios se podrían ubicar en el último cuarto del siglo $X X$, describe una estructura social en transformación que caracteriza al capitalismo tardío, un momento histórico en el que pierde sus componentes centrales y configura una sociedad postindustrial, cuyo paradigma principal de la desigualdad social ya no es el reparto y la distribución de la riqueza socialmente producida, si no el reparto, la minimización, la canalización de los riesgos generados por el propio proceso modernizador. Pero mientras las situaciones sociales de riesgo -nuevas enfermedades, desempleo y desprotección estatalintensifican las diferencias entre el primer mundo y los países periféricos, los riesgos del deterioro ecológico o de desastres atómicos son supranacionales y terminan afectando también a quienes los producen o se benefician de ellos; son daños irreversibles que poseen la capacidad de destruir la vida misma sobre la Tierra.

Este recorrido por el pensamiento de los principales polemistas del tema de la postmodernidad, destaca esencialmente la tendencia globalizadora de los cambios tecnológicos, económicos, culturales y políticos que han dado lugar al surgimiento de un orden social nuevo, en el cual se rompe el correlato emancipador de la modernidad, radicalización que ha generado un estado postnacional, una 
sociedad postindustrial y una cultura postmoderna. En suma, una modernidad distinta -megamodernidad- signada por la aceptación del riesgo en el pacto social, la convivencia con la crisis ecológica, política y social, así como, la dominación completa del capital en la sociedad, ante cuyo -aparentemente ineluctable- desenvolvimiento se requiere no el abandono de la razón y de la ciencia sino la reflexión (auto confrontación) sobre sus problemas y deficiencias, para generar nuevos ámbitos de participación democrática en la toma de decisiones políticas.

\section{De la sociedad del riesgo a la sociedad sostenible}

Las fuerzas del mercado, los cambios tecnológicos y los cambios culturales han venido configurando una sociedad cosmopolita global, una sociedad megamoderna, en la cual se producen riesgos sociales y peligros ecológicos inherentes al desenvolvimiento mismo de sus dimensiones institucionales, generando un progreso autodestructivo que cuestiona su propia racionalidad basada en la ciencia, el derecho y la democracia, y trasciende los límites geográficos y de regulación política del estado nacional. No obstante, sin abdicar de los fundamentos y valores de la modernidad, tanto el sujeto reflexivo de Giddens como la modernidad reflexiva de Beck apuntan al tránsito ineludible -puesto que la humanidad quiere sobrevivir- de la "sociedad del riesgo" a la "sociedad sostenible", en la cual no hay vuelta posible hacia una sociedad edénica, porque no pueden borrarse los adelantos técnicos alcanzados.
Una reflexión crucial en esta dirección es precisamente la forma de generar conocimiento sobre el desarrollo sostenible, en tanto paradigma emergente de la megamodernidad, el cual es asumido por Marín (2010:51) como “...un producto de la evolución en los sistemas humanos (realidad naturaleza-sociedad-economía-género-riesgos: integrada y dinamizada por los hombres y mujeres, así como sus relaciones)...", una realidad compleja que conlleva abordajes interdisciplinarios y transdisciplinarios, es decir, la interacción, integración y complementariedad entre las diversas áreas o disciplinas del conocimiento, a los fines de identificar categorías comunes de análisis que expliquen el comportamiento sistémico y multidimensional del desarrollo sostenible, y consecuentemente, fundamenten su planificación y gestión. En este ámbito se encuentran los enfoques que promueven una visión no convencional del desarrollo y hasta una nueva filosofía científica, tales como conocimiento integrado (Novo, 2003), sistémico (Bossel, 1999) y de la complejidad (Jiliberto $(2002,2003)$.

De acuerdo con Novo (2003), dado que la sociedad de la globalización es un mosaico de graves desequilibrios territoriales, económicos y humanos, se requiere una nueva filosofía del desarrollo, un nuevo esquema de pensamiento integrado capaz de interrelacionar claves éticas, culturales y científicas con claves socioeconómicas, que permita una comprensión de los problemas del medio ambiente en su contexto local, pero imbricados en la escala global. En el terreno de los valores, implica abandonar la postura de 
dominación indiscriminada de la naturaleza, lo cual significa el abandono del antropocentrismo y el etnocentrismo que divide a la sociedad global en ricos y pobres, el respeto por la diversidad biológica, dado su valor intrínseco para el mantenimiento equilibrado de la vida, y el respeto de la diversidad cultural, como elemento de primer orden para el desarrollo de la vida humana a lo ancho del planeta.

En un aporte desde la economía socioecológica, Quiroga (2003) destaca que la sustentabilidad de cualquier actividad humana estaría determinada por la relación entre las dinámicas de crecimiento económico, que insumen materiales y energía, y de resiliencia del ecosistema concreto en lo histórico-espacial, de modo que desarrollar sustentabilidad local, y global, implica redistribuir el acceso al patrimonio natural y la carga ecológica planetaria, tanto en la extracción de recursos como en la producción de desechos. Ello significa reconstruir estilos de vida, y consecuentemente, estilos de de- sarrollo, más sustentables, y avanzar en mejores tecnologías que minimicen el impacto ambiental de las actividades económicas.

En el centro de los enfoques mencionados se encuentra la necesidad de una nueva comprensión de las relaciones sociedad-naturaleza, que permita respetar los umbrales de crecimiento de los sistemas sociales en función de los límites físicos que oponen los ecosistemas, pues no es posible el crecimiento indefinido dentro de una biósfera finita. El énfasis de esta racionalidad viene dado por tres conceptos aportados por la ecología: capacidad de carga $^{1}$, huella ecológica ${ }^{2}$ y resiliencia ${ }^{3}, y$ por una dialéctica global local, dado que los procesos ecológicos son invariantes $\mathrm{y}$, por tanto, independientes de la escala.

En un enfoque sistémico holístico, Bossel (1999) plantea que la sostenibilidad es un concepto dinámico resultado imprevisible de un proceso evolutivo societal ante los cambios ambientales, tecnológicos y culturales, en valores y aspi-

1 Número máximo de individuos de una población de una especie dada que puede ser mantenida en un territorio concreto de manera indefinida, sin que se produzca una degradación en la base de recursos que pueda significar una reducción de la población en el futuro (Riechmann, 1995). En cuanto a la especie humana, esta capacidad de sustentación sólo tiene sentido en relación a todo el planeta, depende del nivel tecnológico y la organización social en un estadio histórico dado.

2 Superficie demandada por una cierta comunidad humana para producir los recursos que consume y absorber los residuos generados, donde quiera que se encuentre esta superficie (Wackernagel y Rees, 2001). A mayor consumo más grande la huella ecológica o demanda sobre la capacidad de carga del planeta, de modo que si las demandas exceden la producción ecológica, disminuirá el capital natural para la actual y las futuras generaciones.

3 Capacidad de un sistema para absorber perturbaciones y reorganizarse, mientras experimenta cambios para conservar esencialmente la misma función, estructura, identidad y reacciones. (Walker et al, 2004). La sustentabilidad, por ende, es la capacidad de un sistema complejo de mantenerse en el tiempo a pesar de la volatilidad ambiental, fomentada por el aprendizaje, la transformación, la renovación y la evolución continua. 
raciones, que se suceden continuamente, proceso que no puede ser planificado ni predicho. En su perspectiva, la sociedad humana es un sistema complejo adaptativo incrustado en otro sistema complejo adaptativo -el ambiente naturaldel cual depende para sostenerse, los cuales coevolucionan en interacción mutua, y cada uno de ellos consiste en un conjunto de subsistemas que también coevolucionan. Consecuentemente, pese a la incertidumbre de la dirección del desarrollo sostenible, es preciso identificar los sistemas esenciales componentes y definir cuáles son los indicadores confiables sobre la viabilidad de cada uno, y del sistema total, siendo la parte crucial de este proceso la identificación y comprensión de las "relaciones esenciales" en el sistema.

El modelo parte de la premisa de que frente a las exigencias o restricciones del ambiente natural, los sistemas que los habitan deben desarrollar ciertas capacidades para poder progresar en ellos, las cuales son los respectivos "orientadores" básicos de viabilidad, que representan preocupaciones o intereses relevantes de la gente y no pueden ser medidos directamente. Entonces, para cada uno de los sistemas y subsistemas que integran una sociedad local particular $y$, dentro de ellos, para cada orientador, es que deben proponerse "indicadores", cuyo objeto es proporcionar toda la información esencial sobre la viabilidad de un sistema y su tasa de cambio, e indicar la contribución al objetivo del sistema total (en este caso, el desarrollo sostenible).

Por su parte, Jiliberto $(2002,2003)$ asume una ontología sistémica donde no tiene cabida la dicotomía sociedad-natu- raleza, por cuanto el mundo fenomenológico -que aparenta estar poblado por entes disjuntos y autónomos de su entornoes de facto un continuum, por lo tanto, ontológicamente una única totalidad, esto es, no hay sistemas, hay un único sistema, un mundo con interexistencia global, donde la única realidad es esta totalidad indivisible e incognoscible, intrínsecamente interdependiente. Acorde con la integralidad que subyace al desarrollo sostenible, propone una epistemología heurístico-enactiva-participativa, en la cual las descripciones no se fundan en lenguajes analíticos, el sistema cognoscente enactúa un mundo que surge de un acto integrado de conocimiento y acción, y el sujeto se halla inscrito de lleno en la descripción, dando lugar a un conocimiento de utilidad contingente.

En términos prácticos significa, primero, asumir que la sostenibilidad local se debe describir ex-novo y con instrumentos de conocimiento propios, que permitan la construcción de un discurso en lenguaje natural acerca de lo que esa sociedad entiende como la totalidad que ella es, mediante la identificación de los elementos centrales de ese sistema, sus lógicas de relación, puntos de conflicto y grados de relevancia, configurando una visión de conjunto que puede haber sido alimentada por el conocer teórico abstracto pero sobre todo por la experiencia acumulada en esa sociedad local. Y segundo, otorgar a esa protodescripción el estatus epistemológico que le corresponde como centro de referencia del proceso de conocimiento y de decisión orientado hacia la sostenibilidad.

Los planteamientos anteriores, no obstante sus diferencias ontológicas y epistemológicas, ofrecen una visión sisté- 
mica, multidimensional y compleja de la sociedad y de la naturaleza, en una dinámica global-local y una lógica contingente, que modifica sustancialmente el discurso del desarrollo surgido en la modernidad, así como la episteme de toda una época. Ya no se trata del anhelo de progreso sustentado en el dominio ilimitado de la naturaleza, tampoco del crecimiento económico irrestricto al margen de consideraciones éticas, ni mucho menos de su estudio desde campos disciplinares estancos. Se trata de que, como condición necesaria para su viabilidad y sostenibilidad en el tiempo, un sistema social concreto en lo histórico espacial debe cambiar y evolucionar, manteniendo su integridad, en interexistencia con el mundo exterior, o sea, debe lidiar con su sistema ambiental cambiante, el estudio de cuyos procesos complejos conlleva aproximaciones interdisciplinarias y transdisciplinarias.

\section{Conclusiones}

A partir de la discusión realizada, se puede intentar una aproximación a los elementos que describen fenomenológicamente el desarrollo, en tanto discurso de la actual megamodernidad, cuya preeminencia de las fuerzas del mercado, el control social, el poderío militar y el industrialismo, genera riesgos económicos, sociales, políticos y ambientales, de alcance planetario, los cuales han demostrado ser difíciles de abordar desde la apreciación onto-epistemológica de nuestra civilización, que se asume como especie separada de la naturaleza y a ésta como objeto exterior, el cual puede ser conocido de manera "objetiva" analizando sus infinitas partes e integrando después el todo.
Así, el desarrollo (nacional, regional, local) sería el proceso que llevan a cabo las sociedades, en un particular ámbito espacial, histórico y cultural, en procura de su bienestar, a través de patrones complejos de interrelación entre los diversos actores o agentes sociales, utilizando, mejorando e incrementando los recursos materiales e inmateriales del territorio, y bajo el requerimiento de articular, o armonizar, las dimensiones de la realidad en que tienen lugar sus fenómenos, comportamientos y actividades, para permitir la sustentabilidad, continuidad, adaptación, evolución y durabilidad del proceso mismo de desarrollo.

Este reconocimiento del comportamiento complejo del desarrollo sostenible amerita una ampliación de la racionalidad clásica, donde la realidad ya no está dada sino que es inter-subjetiva, por lo cual requiere la praxis reflexiva del sujeto, es decir, la incorporación del observador, con su andamiaje axiológico, en el proceso de observación, con metodologías que permitan aprehender sus propiedades y enfocar los problemas no sólo como problemas complejos en sí mismos, sino además como inseparables y complementarios.

\section{Referencias bibliográficas}

Beck, Ulrich (1994). La sociedad del riesgo. Hacia una nueva modernidad. Barcelona, Paidós.

Beck, Ulrich (2003). Sociedad y desarrollo. La sociedad del por qué no. Tareas, $\mathrm{N}^{\circ}$ 115: 97-104, Cela, Panamá. Disponible: http://168.96.200.17/ar/libros/ tar115/beck.rtf (consulta: mayo15, 2010).

Beltrán, Miguel; Cardona, Marleny (2005). La sociología frente a los espejos del 
tiempo: Modernidad, postmodernidad y globalización. Medellín, Universidad EAFIT, Cuaderno de Investigación $\mathrm{N}^{\circ} 28$.

Berman, Marshall (1991). Todo lo sólido se desvanece en el aire. La experiencia de la modernidad. Bogotá, Siglo XXI Editores, e. 5.

Bossel, Hartmut (1999). Indicators for sustainable development: Theory, method, applications. A Report to the Balaton Group. Winnipeg, Canada, International Institute for Sustainable Development (IISD).

Carretero, Angel (2002). Postmodernidad y temporalidad social. A Parte Rei. Revista de Filosofía, $N^{\circ} 24$, Universidad Complutense de Madrid. Disponible: http://serbal.pntic.mec.es/ cmunoz 11/cilea.pdf

Giddens, Anthony (1994). Consecuencias de la modernidad. Madrid, Alianza.

Giddens, Anthony (2002). Modernidade e identidade. Traducción de Plínio Dentzien. Rio de Janeiro, Zahar, Jorge (ed.).

Habermas, Jürgen (1989). Modernidad: un proyecto incompleto. En: Casullo, Nicolás (ed.), El debate Modernidad Pos-modernidad, Buenos Aires, Editorial Punto Sur.

Habermas, Jürgen (1992). Teoría de la acción comunicativa. Buenos Aires, Taurus.

Jiliberto, Rodrigo (2002). Una mente pródiga para un mundo sistémico. Disponible: www.culturasostenible.com (consulta: enero 09, 2011).

Jiliberto, Rodrigo (2003). Apuntes tentativos para una epistemología de la sostenibilidad. Conferencia, Instituto de Gobernabilidad, Barcelona, España. Disponible: www.culturasostenible. Com/mostrarDocumentPdf.php?id $=15$ (consulta: enero 10, 2011).
Lechner, Norbert (1999). El Estado en el contexto de la modernidad. En: Lechner, Norbert; Millán, R.; Valdés, F. (coord.). Reforma del Estado y coordinación social, México, Plaza y Valdés Editores.

Lyotard, Jean-Francois (1987). La condición postmoderna. Informe sobre el saber. Buenos Aires, Ediciones Cátedra.

Marín, Freddy (2010). Construcción de conocimiento sobre desarrollo sostenible desde una perspectiva inter y transdisciplinaria. Investigación en Ciencias Humanas, Vol. 1, Venezuela, Universidad del Zulia, pp 49-68.

Novo, María (2003). El desarrollo sostenible: sus implicaciones en los procesos de cambio. Polis, Revista On-Line de la Universidad Bolivariana, $\mathrm{Vol} 1, \mathrm{~N}^{\circ} 5$, Santiago de Chile. Disponible: www.revistapolis.cl/5/ind5.htm (consulta: mayo 27, 2010).

Quiroga, Rayén (2003). Para forjar sociedades sustentables. Polis, Revista On-Line de la Universidad Bolivariana, Vol. $1, N^{\circ} 5$, Santiago de Chile. Disponible: redalyc.uaemex.mx/pdf/305/305005 10.pdf (consulta: mayo 27, 2010).

Riechmann, Jorge (1995). "Desarrollo sostenible: la lucha por la interpretación". En: Riechmann, J. et al. (eds.), De la economía a la ecología, Madrid, Trotta.

Wackernagel, Mathis y Rees, William (2001). Nuestra huella ecológica: Reduciendo el impacto humano sobre la tierra. Santiago de Chile, Editorial Lom.

Walker, Brian; Holling, Crawford; Carpenter, Stephen y Kinzig, Ann (2004). Resilience, adaptability and transformability in social-ecological systems. Ecology and Society, 9(2): 5. [online]. Disponible: http://www.ecologyandsociety.org/vol9/iss2/art5/ (consulta: noviembre 10, 2010). 